\title{
5th Winfocus World Congress, Sydney, Australia, 4-6 September 2009
}

\section{USE OF BEDSIDE ULTRASOUND TO CONFIRM EMERGENCY ENDOTRACHEAL TUBE POSITION - A PILOT STUDY}

B. Hoffmann, J. P. Gullett, H. F. Hill, W. T. Hosek, E. S. Bessman, M. L. McCarthy

Objective: To determine feasibility and accuracy of bedside ultrasound (BUS) for diagnosing endotracheal tube (ETT) position in the emergency setting.

Methods: This prospective, single-blinded study enrolled study subjects into three groups: simultaneous BUS of the neck performed during emergency department (ED) intubation (S), BUS within 3 min of ED intubation (A/ED), BUS within 3 min of patient's ED arrival after pre-hospital intubation (A/EMS). ED providers performing BUS had received standardized ultrasound training, intubators were blinded to ultrasound findings. Cormack and Lehane grades were used to classify each intubation attempt into an "easy", "moderate" and "difficult" category. Additional data collected included diagnostic accuracy of sonographer and intubator compared to clinical outcome, anatomy identified with sonography and time to diagnosis of ETT position for sonographer and intubator.

Results: During a 9-month period, 89 study subjects with a total of 115 intubation attempts were captured, 15 attempts were excluded for incomplete data. In 100 attempts (62-easy, 19-moderate, 19-difficult), sonographers were $100 \%$ accurate determining ETT position, intubators were $98 \%$ accurate compared to clinical outcome.

Blinded quality review of documented sonographic neck anatomy confirmed all BUS findings. An empty esophagus was $100 \%$ specific for correct ETT placement and an esophageal foreign body detection $100 \%$ specific for esophageal intubation.

Sonography time to diagnosis was significantly faster than intubator time to diagnosis in all categories ( $p<0.0001$, "easy", $n=47$; $p=0.007$, "moderate", $n=14 ; p<0.0001$, "difficult", $n=19$; paired $t$ test, A/EMS cases excluded for analysis).

Conclusion: In this study, ultrasound showed an accuracy of $100 \%$ determining ETT position.

\section{THE REAL-TIME OBSERVATION WITH ULTRASONOGRAPHY: IS IT ABLE TO SAVE THE TIME TO CONFIRM ESOPHAGEAL INTUBATION FOR TRAINEES?}

\author{
Youdong Sohn \\ Hallym University Medical Center, South Korea
}

Background: The intubation is one of important skills that trainees have to be competent. The skill of intubation in proper way is not only important, but also the confirmation of intubation properly is mandatory. Secondary confirmation of correct tracheal intubation relies on auscultation, capnography, chest X-ray and so on. One of characteristics of ultrasonography is real-time observation that may save the time to confirm the correct tracheal intubation. In this study, we investigated whether the use of ultrasonography during intubation is helpful for trainees to confirm the correct tracheal intubation.

Methods: Eight patients required tracheal intubation with ultrasonography were studied. To compare the study group, we collected thirty three patients who had been intubated without ultrasonography before the study period. The outcome was the interval time from the holding of tracheal tube to secure the tube with tape finally. During this investigation, the high frequency linear transducer was put on the left lateral side of mid-neck transversally to observe the esophageal opening with tube. We recorded a failure as esophageal intubation at first attempt.

Results: The mean age, number of male, frequency of esophageal intubation and the interval time until final intubation showed no significant difference between two groups. To evaluate the intervaltime difference of between subjects, two-way ANOVA was performed. It showed a statistical significance between two groups, status (success vs. failure), and interaction (group $\times$ status). According to Kaplan-Meier survival analysis, the study group showed shorter time to correct the esophageal intubation with a significant difference statistically [217 $\mathrm{s} \pm 41$ (SE) vs. $579 \mathrm{~s} \pm 157$ (SE), $p=0.034$ ]. Conclusion: This study describes that the real-time observation with ultrasonography for trainees saves time to confirm the tracheal intubation.

\section{SONOGRAPHIC IDENTIFICATION OF TUBE THORACOSTOMY STUDY (SITT STUDY)}

Jamie Goodis, Stephanie Doniger, Scott Bradley, Sarah Williams, Laleh Gharahbaghian, Steve Crandall, David Spain

Stanford University School of Medicine, Stanford, CA, USA

Objectives: Thoracostomy tubes are commonly placed in the management of surgical, emergency, and trauma patients. Chest radiographs and CT scans are performed to localize them. Our goal is to develop a novel approach to utilize bedside ultrasonography to identify intra-thoracic chest tube placement.

Methods: This was an IRB-approved observational study, with a convenience sampling of patients with previously placed chest tubes. Bedside ultrasound was performed using the $5-1$ and $10-5 \mathrm{MHz}$ transducers, in both 2D and M-modes. The chest tubes were identified in transverse and sagittal views by starting at the skin entry point and scanning to the point at which the chest tube penetrated the pleural line. All ultrasounds were reviewed by fellowship-trained attending physicians. Tube placement was compared with the gold-standard of chest radiographs.

Results: Nine patients with 10 chest tubes were enrolled. Chest tubes were visualized as entering the pleural space in $100 \%(10 / 10)$. This was best visualized with the $10-5 \mathrm{MHz}$ linear probe. In six patients, M-mode was preformed along the presumed chest tube and adjacent 
structures. Characteristic M-mode patterns distinguished the chest tube from adjacent ribs and the pleural line.

Conclusions: Our study suggests that bedside ultrasound can localize the entrance of the tube into the thoracic cavity as well as distinguish it from ribs, pleural line by characteristic M-mode patterns. This is best visualized with the $10-5 \mathrm{MHz}$ linear probe. This is a novel approach that can lead to decreased radiation exposure as well as expedite care of critically ill patients.

\section{IMPACT OF IMAGE PROCESSING ON THE PLEURAL SLIDING SIGN}

\section{Michelle Holm}

\section{Hennepin County Medical Center, Minneapolis, MN, USA}

Study objective: Sonographic identification of pleural sliding is a quick and simple means to rule-out pneumothorax. Evaluation for pneumothorax is now a routine part of the FAST (Focused Assessment with Sonography for Trauma) exam. Modern ultrasound machines use several image processing features to improve image quality, and all of these features are typically activated during a FAST exam. We evaluated the combined effect of tissue harmonics, SonoMB ${ }^{\mathrm{TM}}$ (spatial compounding) and SonoHD ${ }^{\mathrm{TM}}$ processing on the identification of pleural sliding.

Methods: This was a prospective study in which 25 physicians were asked to evaluate for pleural sliding in a healthy male. Using a SonoSite M-Turbo with a curved array transducer each physician scanned the right side of the chest in the 3rd to 4th intercostal space in the mid-clavicular line. Each physician was asked to look for pleural sliding with the image processing features in two different configurations: with tissue harmonics, SonoMB ${ }^{\mathrm{TM}}$ and SonoHD ${ }^{\mathrm{TM}}$ activated, then with all three of these features deactivated. Physicians were then asked if it was easier to see pleural sliding with all of these features on or off, or if it made no difference.

Results: $23 / 25$ physicians (92\%) thought it was easier to see pleural sliding with all image processing features deactivated, 1/25 thought it was easier to see pleural sliding with all image processing features activated and $1 / 25$ saw no difference $(p<0.001)$.

Conclusion: Some processing features that improve abdominal and cardiac imaging make it more difficult to recognize pleural sliding. To our knowledge, this is the first study of the impact of image processing on the pleural sliding sign.

\section{THROMBOEMBOLIC EVENTS DURING VENOUS COMPRESSION ULTRASOUND OF THE LOWER EXTREMITY IN PATIENTS WITH DEEP VENOUS THROMBOSIS}

\author{
Srikar Adhikari ${ }^{1}$, Wes Zeger $^{1}$, Llir Frrokaj ${ }^{1}$, Michael Blaivas ${ }^{2}$ \\ ${ }^{1}$ University of Nebraska Medical Center, Omaha, NE, USA \\ ${ }^{2}$ Northside Hospital-Forsyth, Atlanta, GA, USA
}

Objectives: Physicians are increasingly utilizing venous compression ultrasound in the evaluation of lower extremity for deep venous thrombosis (DVT). Focused Compression ultrasound has proven to be highly sensitive and specific for identifying DVT. Few case reports have been published describing the occurrence of pulmonary embolism caused by dislodging a DVT during compression ultrasound. To our knowledge, no prior studies investigated the risk of thromboembolic events during venous compression ultrasound. The objective of this study is to determine the risk of thromboembolic events during compression ultrasound in patients with DVT.

Methods: This was a retrospective review of all Emergency Department (ED) patients who underwent venous compression ultrasound of the lower extremity for evaluation of DVT over a 6 year period. This study took place at an academic urban ED with an annual census of 48,000 visits. The ultrasound protocol included B-mode and Doppler color flow analysis of deep veins of lower extremity along with compression of common femoral, superficial femoral, deep femoral, popliteal, posterior tibial, peroneal, and greater saphenous veins. Three chart reviewers performed data collection using a standardized data extraction form. A systematic review of medical records was accomplished for patients diagnosed with DVT. Presence of any one of these clinical features is used to identify a thromboembolic event: new onset or worsening shortness of breath, chest pain, palpitations, syncope, hypotension, hypoxia and death within $24 \mathrm{~h}$ after compression ultrasound. Descriptive statistics are used to analyze the data. Continuous data are presented as means with standard deviations and dichotomous data are presented as percent frequency of occurrence with $95 \%$ confidence intervals. Inter-observer agreement among chart reviewers was assessed by kappa analysis.

Results: A total of 2,451 patients (1,595 females, 856 males) were identified over a six-year period. The mean age of the patients was 60 years \pm 19 (SD). Three patients were excluded from the analysis due to missing data. Inter-observer agreement among chart reviewers was high $(k=0.90)$. DVT was identified in $363(15 \%$, CI $13-16 \%)$ patients. Common femoral vein was most commonly involved $(77 \%$, CI $72-81 \%) .45 / 363$ (12\%, CI 9-15\%) were noted to have chronic DVT. No thromboembolic events occurred from compression ultrasound in patients with DVT. All patients with acute DVT were discharged on anticoagulation therapy.

Conclusions: There was no evidence of thromboembolic events from venous compression ultrasound in our study. Risk of pulmonary embolism from compression ultrasound is extremely low.

\section{ISOLATED DEEP FEMORAL VEIN THROMBOSIS: IMPLICATIONS FOR PHYSICIAN PERFORMED 2-POINT COMPRESSION ULTRASONOGRAPHY}

Srikar Adhikari, Wes Zeger, Llir Frrokaj

University of Nebraska Medical Center, Omaha, NE, USA

Objectives: 2-point compression ultrasonography is widely utilized by physicians to evaluate lower extremity for Deep Venous Thrombosis (DVT). 2-point compression technique includes compressing common femoral vein and popliteal vein. Despite a lack of definite evidence, some recommend to compress additional sites such as deep femoral vein and superficial femoral vein. The objective of this study is to determine the validity of a limited 2-point compression technique and distribution of deep venous thrombi in the lower extremity.

Methods: This was a retrospective review of all Emergency Department (ED) patients who underwent venous ultrasound imaging of the lower extremity for evaluation of DVT over a 6 year period. This study took place at a tertiary care academic ED with an annual census of 48,000 visits. The ultrasound protocol included both B-mode and Doppler color flow analysis of deep veins of lower extremity. A total of three chart reviewers performed data collection using a standardized data extraction form developed using Microsoft Access. Data extraction form included information about ultrasound findings, final interpretation, ED assessment, additional diagnostic testing, hospital course, outcome and final diagnosis. Data are summarized using descriptive statistics. Continuous data are presented as means with standard deviations and dichotomous data are presented as percent frequency of occurrence with $95 \%$ 
confidence intervals. Inter-observer agreement among chart reviewers was assessed by kappa analysis.

Results: A total of 2,451 patients (female 1,595, male 856) underwent lower extremity ultrasound examinations. The mean age of the patients was 60 years \pm 19 (SD). Inter-observer agreement among chart reviewers was high $(k=0.95)$. DVT was detected in $363(15 \%$, CI $13-16 \%)$ patients. Thrombus confined to common femoral vein was found in $3(0.83 \%, \mathrm{CI}-0.1$ to $1.7 \%)$ cases. Isolated Popliteal vein thrombus was identified in $17(4.6 \%$, CI $2.5-6.8 \%)$ cases. Isolated calf vein thrombi were found in 58 (16\%, CI 12-19\%) patients. Thrombus in the superficial femoral vein alone was identified in 8 (2.2\%, CI $0.69-3.7 \%)$ patients. Isolated Deep Femoral vein thrombus was identified in only $1(0.28 \%$, CI -0.26 to $0.8 \%)$ patient. Chronic DVT were found in 45/363 (12\%, CI 9-15\%) patients. D-dimer was not obtained in all patients with DVT. D-dimer was found to be elevated in patient with isolated deep femoral vein thrombosis.

Conclusions: Isolated deep femoral vein thrombi are extremely rare. Our study results do not support addition of deep femoral vein compression to the 2-point compression ultrasonography technique.

\section{APPLICATION OF ULTRASOUND FOR HEMOBILIA: A CASE REPORT ON A RUPTURED ANEURYSM OF THE HEPATIC ARTERY}

Kouji Oh, Ken Suzawa, Masaaki Ogata

Kobe City Medical Center West Hospital, Japan

Presented is a case of 48-year-old man who was referred for severe precordial and epigastric pain. The symptom diminished in 20 minutes without any prescriptions, however, repeated paroxysmally. The blood test showed leukocytopenia, slight anemia and the elevation of serum amylase, AST and ALT. Ultrasound showed the swollen pancreas as well as debis-like contents in the gallbladder. The common bile duct was visualized as a hypoechoic duct instead of a sonolucent appearance. The following computed tomography (CT) presented the similar findings. The initial diagnosis was made as acute edematous pancreatitis on the basis of the ultrasound and CT images as well as the laboratory data. After admission, endoscopy revealed biliary bleeding via the duodenal papilla. Endoscopic retrograde cholangiography demonstrated a spindle-shaped filling defect in the common bile duct. On the follow-up examination with ultrasound, a cystic lesion of $25 \mathrm{~mm}$ in diameter appeared in the liver. Color Doppler demonstrated that the lesion had swirling flow in it and was enhanced with perflubutane. An aneurysm ruptured to the biliary tract was supposed to cause hemobilia. Emergency angiography confirmed it as a ruptured aneurysm of the right hepatic artery in the liver, and then transcatheter arterial embolization was successfully performed, and consequently, hemobilia was healed. Although hemobilia is rare in the gastrointestinal hemorrhage, it is critically important to identify the evidence of hemobilia as well as the causative lesion. Ultrasound as well as color Doppler can be available for the purpose, and may present significant findings for hemobilia.

\section{ULTRASONOGRAPHIC IMAGING FEATURE IN ACUTE CHOLANGITIS WITHOUT DISTAL BILIARY OBSTRUCTION}

Hee-Jin Kwon, Kyung-jin Nam, Jin-han Cho

Dong-A University Hospital, Seoul, Korea

Purpose: The aim of this study is to differentiate the cause of acute cholangitis without biliary obstruction.
Materials and methods: A total of 81 patients who had acute cholangitis as identified on clinical setting were retrospectively included in this study. Patients were divided into two groups: patients with biliary obstruction such as stone, tumor, lymph node, stricture and patents without biliary obstruction. First group are excluded in this study. A total of 27 patients $(33 \%)$ had acute cholangitis without definite distal biliary obstruction. We analyzed the cause, prognosis, imaging feature with USG.

Results: The cause of acute cholangitis without biliary obstruction were active C.S. cholangitis in 11 cases $(40.7 \%)$, post operative swelling after gastrojejunostomy, PPPD, choledochojejunostomy $(22.2 \%)$, Mirizzi syndrome in 1 case $(3.7 \%)$, recent passage of distal CBD stone in 2 cases $(7.4 \%)$, primary sclerosing cholangitis in 2 cases(7.4\%), compression effect by large duodenal diverticulum in 1 case $(3.7 \%)$, and unknown case in 4 cases $(14.8 \%)$

Conclusion: Acute cholangitis may be associated without distal biliary obstruction. Ultrasonographic image can provide more precise information about cause of acute cholangitis and help to differentiate.

\section{THE CONTRIBUTION OF ULTRASONOGRAPHY TO THE DIAGNOSTIC THERAPEUTIC TRIAGE OF THE BILIO-PANCREATIC EMERGENCIES}

\author{
Adela Golea, Radu Badea, Titus Suteu, Lidia Ciobanu, \\ Teodora Surdea-Blaga
}

University of Medicine, Romania

Objectives: The study proposes the evaluation of the emergency ultrasonography role ( $24 \mathrm{~h}$ of 24$)$ in the diagnostic-therapeutic triage of the bilio-pancreatic emergencies.

Materials and methods: In the Emergency Department there was ultrasonographicaly evaluated a number of 351 patients, aged between 18 and 90, during 6 December 2005-6 June 2006. It was analyzed the contribution of the ultrasonography to the diagnostic triage of the patients and it was established the adequate emergency therapy.

Results: Analyzing correlatively the diagnostic contribution of the ultrasound examination in emergency in the case of the patients who came with the clinical characteristics of biliary colic, we can observe the improvement of the curative decision with the objective selection of the patients who can be sent home and of the indication of immediate surgery in $45.8 \%$ of the cases. Regarding the icterus syndrome, it can be observed that the emergency ultrasound examination is an important element for the differentiation of the obstructive icterus from the hepatocellular one.

Discussion: The study presents an analysis of the contribution of ultrasonography to the etiology evaluation of the clinical characteristics of biliary emergency manifestations. The statistical analysis of the logistic regressive type, applying the Odds Ratio test showed that the use of ultrasonography as a selection method of the patients arrived with biliary colic helps significantly to the detection of complications and implicitly determines the increase of the number of patients hospitalized for complications of the biliary lithiasis (odds ratio $=9.87$ )

Conclusions: The ultrasonography is the examination of first intention in emergency having a role in the diagnostic triage of the patients with severe bilio-pancreatic symptomatology. The ultrasound examination allows the immediate curative decision (immediate surgery/ intensive care) or the guidance towards other specialized services for supplementary investigations. 


\section{ROLE OF EMERGENCY ULTRASOUND IN THE} DETECTION AND EARLY TREATMENT OF NONHODKING'S DISEASE PRESENTING AS ABDOMINAL PAIN AND OBSTRUCTIVE JAUNDICE: A CASE REPORT

\author{
Matteo Frameglia \\ Hospital Sacro Cuore-Don Calabria, Italy
}

We reported a 81-year-old man case presented to Emergency Department with right hypocondrial pain and jaundice.

In many cases emergency ultrasound of the right upper quadrant may be diagnostic for biliary disease, or may identify alternative causes of the patient's symptoms. Emergency ultrasound also helps to distinguish emergent, urgent and expectant conditions.

In our case, the emergency ultrasound, performed by emergency physician, demonstrated dilated intrahepatic and extrahepatic biliary ducts, hepatosplenomegaly, parahortic, hilar and celiac lymphadenopathy and absence of cholelitiasis.

Percutaneous aspiration cytology of the hilar mass confirmed the diagnosis of non Hodgkin's lymphoma.

The combination of percutaneous transhepatic biliary ducts drainage with systemic chemotherapy was extremely effective in our patient. The patient still lives with no evidence of disease after 24 months of follow up.

The value of ultrasound examination in the differential diagnosis of jaundice is emphasized.

While other examinations provide information that is more detailed, emergency ultrasound is non-invasive, is rapidly deployed and does not entail removal of the patient from the emergency area. Further avoids the delays, costs, the administration of contrast agents and radiations.

\section{BEDSIDE ULTRASOUND IS USEFUL FOR DIAGNOSING COLONIC DIVERTICULITIS IN THE EMERGENCY DEPARTMENT}

Kuo-Chih Chen, Liang-Ting Wu, Chee-Fah Chong,

Tzong-Luen Wang

Emergency Department, Shin-Kong Wu Ho-Su Memorial Hospital, Taiwan

Objectives: The aim of this study was to investigate the role of bedside ultrasound performed by emergency physicians for diagnosis of colonic diverticulitis.

Methods: We retrospectively reviewed medical records from 101 adult patients with a diagnosis of colonic diverticulitis in the emergency department from 1 January 2007 to 31 December 2008. All of the patients were confirmed by computed tomography. Sixteen patients received bedside ultrasound before undergoing computed tomography and were enrolled in this study. All the bedside ultrasound scans were performed by one experienced emergency physician.

Results: Of the 16 patients underwent bedside ultrasound survey with a final computed tomography diagnosis of colonic diverticulitis, we correctly diagnosed colonic diverticulitis in 15 patients (93.8\%). The locations of the lesion were ascending colon (73.3\%), descending colon $(13.3 \%)$, transverse colon $(6.7 \%)$, and cecum $(6.7 \%)$. The most common sonographic finding was inflamed colonic wall with segmental thickening (100\%); other findings included dome sign $(80.0 \%)$, ascites $(13.3 \%)$, and abscess formation $(6.7 \%)$. The mean and median time for sonographic diagnosis of diverticulitis were 126 and $105 \mathrm{~min}$, compared with 252 and $217 \mathrm{~min}$ for computed tomography diagnosis.
Conclusions: The present study shows that emergency physicians can diagnose colonic diverticulitis by bedside ultrasound with sufficient accuracy. Moreover, significantly faster diagnosis by bedside ultrasound compared to computed tomography also provides prompt treatment and disposition for the patients.

\section{THE SIGNIFICANCE OF ULTRASOUND IN DECISION- MAKING OF SURGICAL INTERVENTION FOR ACUTE COLONIC DIVERTICULITIS}

Ken Suzawa, Masaaki Ogata, Kouji Oh

Kobe City Medical Center West Hospital, Japan

To evaluate the significance of ultrasound in decision-making of surgical intervention for acute colonic diverticulitis, we reviewed the relation of sonographic appearances with surgical indications in 51 cases of the entity examined with ultrasound at admission during the latest 5 years. Thirty-eight cases were relieved with conservative treatments and 13 cases underwent laparotomy in the acute or subacute phase of the entity. The surgical indications were possible acute appendicitis in 5 , diffuse peritonitis in 4 , persistent abscess in 3 and large bowel obstruction in one. Ultrasound directly presented gasechoes penetrating through the thickened colonic wall in one case of diffuse peritonitis, a localized abscess in 9, and large bowel obstruction at the lesion in one. Regional wall-thickening of the colon was the most common ultrasonographic appearance, followed by surrounding hyper-echoic connective tissues (omentum or mesenterium). Diverticula were recognized as gas-echoes in the thickened wall or protruded colonic wall with fecolith. On the other hand, ultrasound showed no significant abnormalities in three and presented a false diagnosis as acute appendicitis in three and cholecystitis in one. In four cases, it was difficult to discriminate diverticulitis from colon cancer on ultrasonograms. Ultrasound was helpful to demonstrate significant findings for decision-making of surgical intervention for acute colonic diverticulitis.

\section{ACUTE APPENDICEAL DIVERTICULITIS}

Tadao Kubota ${ }^{1}$, Koichiro Kubo ${ }^{2}$, Yoshihiro Morimoto ${ }^{2}$, Kei Hasegawa ${ }^{2}$

${ }^{1}$ Department of Surgery, Okinawa Hokubu Hospital, Japan

${ }^{2}$ Department of Surgery, Chibanishi General Hospital, Japan

Appendiceal diverticulitis is rare clinical entity with an incidence of under $1 \%$ of all acute appendicitis. Preoperative images of acute appendiceal diverticulitis are rarely reported because of the difficulty of distinguishing appendiceal diverticulitis and other iliocecal diseases like acute appendicitis or cecal diverticulitis.

We report a case of preoperative diagnosed acute appendiceal diverticulitis. A 30-year-old female with a presumptive diagnosis of acute appendicitis from history and physical examination was admitted to our hospital. Ultrasound sonography showed inflamed appendiceal diverticula and inflammatory changes of the surrounding tissue. She underwent emergent appendectomy and pathological specimen revealed inflammatory changes of diverticula within the appendix.

This is a case of preoperative diagnosis of acute appendiceal diverticulitis with abdominal ultrasound. Careful examination with ultrasound can distinguish form appendiceal diverticulitis from appendicitis or other iliocecal disease. 
The swollen appendix was detected but its findings were slightly different from that of typical acute appendicitis in the following points. One difference was the thickened wall of the appendix. The other difference was the presence of air inside of the appendix.

\section{EMERGENCY PHYSICIANS CAN ACCURATELY DIAGNOSE ADULT INTUSSUSCEPTION IN THE EMERGENCY DEPARTMENT}

Kuo-Chih Chen, Te-Hao Wang, Chee-Fah Chong, Tzong-Luen Wang

Emergency Department, Shin-Kong Wu Ho-Su Memorial Hospital, Taiwan

Objectives: Intussusception is rare in adult patients. The ultrasound has been a useful tool for pediatric intussusception. The aim of this study was to investigate whether bedside ultrasound performed by emergency physicians can assist diagnosis for adult intussusception in the emergency department.

Methods: We retrospectively reviewed 17 medical records from patients older than 18 years old with a diagnosis of intussusception in the emergency department from 1 May 2002 to 31 March 2009. All of the diagnoses were confirmed by computed tomography and/or pathologic findings.

Results: Among these 17 patients, the mean age was 47 years old, ranging from 18 to 90 , and eleven of them were men. The major causes for emergency department visiting were abdominal pain lasting for 3 days (47\%) and abdominal fullness (23\%). The emergency physicians performed bedside ultrasound for 15 patients prior to computed tomography. All of the scans revealed pseudo-kidney or target sign. The causes for adult intussusception were lipoma in eight patients, adenocarcinoma in three patients, lymphoma in two patients, hamartomatous polyp in one patient, and ectopic pancreatic tissue with polyp formation in one patient. Two patients refused surgery.

Conclusions: Emergency physicians should consider adult intussusception for patients presenting with prolonged abdominal pain and abdominal fullness. The present study shows that emergency physicians can accurately diagnose adult intussusception in the emergency department. This can lead to early surgical consultation and prevent complications.

\section{ULTRASOUND FOLLOW-UP OF ABDOMINAL PAIN IN THE INTESTINAL AND PERITONITIS TUBERCULOSIS TREATMENT}

Maria Goretti Ametembun

Maternity Emma Poeradiredja Hospital, Bandung, Indonesia

Background: This study was conducted at Maternity Emma Poeradiredja Hospital Bandung from 1 January 2007 to 30 April 2009 to follow-up the abdominal pain of the intestinal and peritonitis tuberculosis treatment.

Methods: The intestinal and (dry type) peritonitis tuberculosis patients were examined with transabdominal ultrasound using ALOKAProsound-SSD3500 before, during and after anti-tuberculosis treatment. The small bowel wall thickening was measured on the tympanic area as well as on the affected dullness area according of dam-board phenomena.

Results: 52 patients, 27 (52\%) male, 25 (48\%) female, age of 19-56 (mean 31). Preceding treatment: the mean of small bowel wall thickness on tympanic area was $0.46 \mathrm{~cm}$ otherwise on dullness area was $1.21 \mathrm{~cm}$ (2.63 times more than tympanic area). Moreover, hypoperistaltic movement, differentiation of wall layers was lost, and irregular margin of small bowel wall were found in the affected dullness area. During the 9-12 months of the treatment period, the signs and symptoms, abdominal colic pain, dullness area and small bowel thickening gradually decreased. After the treatment: no colic abdominal pain, no more tenderness, no doughy abdomen nor dullness area anymore. The mean of small bowel wall thickening decreased to $0.51 \mathrm{~cm}$, and normal peristaltic movement were seemed on the affected area, otherwise the margin of the wall was still irregular.

Conclusion: The abdominal pain and the small bowel wall thickening gradually decreased according healing of intestinal and peritonitis tuberculosis.

\section{RECURRENT ABDOMINAL PAIN AMONG THE DISSEMINATED TUBERCULOSIS CASES IN SAUMLAKI COMMUNITY HEALTH CENTRE, TANIMBAR THE REMOTE BOUNDARY ISLANDS IN THE WEST OF SOUTH-EAST MOLUCCAS, INDONESIA}

\section{Maria Goretti Ametembun}

Saumlaki Community Health Centre, Tanimbar Islands, West of South-East Moluccas, Indonesia

Background: This study was conducted at Saumlaki Community Health Centre at Tanimbar, the remote boundary islands in the West of South-East Moluccas (Indonesia) from 20 to 23 January 2009 to know the recurrent abdominal pain among all disseminated tuberculosis out-patients.

Methods: All patients with disseminated tuberculosis (lung tuberculosis, cervical lymphadenopathy, peritonitis tuberculosis and chronic pyelonephritis) were diagnosed base on history and physical examinations by an internal medicine doctor were examined with transabdominal ultrasound (FUKUDA SAN FS906 portable ultrasound machine). No laboratory nor radiology facilities.

Results: 485 patients, including $156(32.2 \%)$ children of $0.5-13$ years (mean 6.2) and $329(67.8 \%$ ) adults of 13-81 years (mean 24.5), 165 (34\%) male, $320(66 \%)$ female. There were $182(37.5 \%)$ patients with the paraaortal lymph-nodes enlargement, $20(4.1 \%)$ with heterogeneous hyperechoic pattern of the pancreas, $352(72.6 \%)$ with irregular margin and hypoechoic pattern of the cortex of the kidney, $1(0.2 \%)$ with pelvocalyectasis without kidney stone, and $31(6.4 \%)$ with irregular thickening of the urinary bladder wall. 485 (100\%) thickening and irregular margin of the small bowel wall were found in all affected dullness area of peritonitis tuberculosis.

Conclusion: In Tanimbar, a remote boundary islands, the abdominal pain within the disseminated tuberculosis patients was related to the small bowel wall thickening according the intestinal tuberculosis, the chronic process of pancreas and kidneys as well as chronic cystitis.

\section{GRAY SCALE AND COLOR DOPPLER SONOGRAPHIC FINDINGS OF THE SPERM GRANULOMA}

Yoo-Dong Won, Seung-Eun Jung, Ki-Tae Kim

Department of Radiology, the Catholic University of Korea, Korea

Objective: The aim of this study was to describe the gray scale and color Doppler sonographic findings of pathologically proven sperm granuloma. 
Materials and methods: We analyzed sonographic findings of twelve cases of pathologically proven sperm granuloma between August 1999 and December 2007. The patients ranged in age from 27 to 68 years (mean, 45 years). Sonographic findings were determined by retrospective analysis of the images. We recorded the size, location, shape, border definition, echogenicity of the lesion, heterogeneity and vascularity. The presence of scrotal swelling and pains were also evaluated.

Results: Eleven of the twelve sperm granulomas were located in the epididymis and one in the seminal vesicle. Our review of the sonographic findings revealed that all twelve lesions had well-defined margins. Five lesions were isoechoic compared with the normal echogenicity of the testis and the remainder of lesions hypoechogeic. In terms of the shape, six lesions show lobulated contour and six masses oval shape. Most of the lesions were heterogeneous on gray scale sonography. On color Doppler sonography, six of lesions showed increased vascularity.

Conclusion: The sperm granuloma shows well-circumscribed heterogeneous iso- or hypo-echoic mass in the epididymis and seminal vesicle. Although only imaging findings cannot lead to diagnose the lesion, knowledge of imaging findings of sperm granuloma can lead to a precise differential diagnosis.

\section{ULTRASONIC DIAGNOSIS OF SUPERFICIAL PATHOLOGICALLY ENLARGED LYMPHNODES}

Liu Mingyu

The Fourth Hospital of Hebei Medical University, China

Objective: To evaluate the significance of ultrasound in diagnosis of the superficial pathologically enlarged lymphnode.

Methods: A prospective study was undertaken with high frequency 2DUS and CDFI in 91 enlarged peripheral lymphnode of 72 patients with different diseases. The sonographic features and vascular peak systolic velocity $\left(V_{\max }\right)$, and diastolic velocity $\left(V_{\min }\right)$, resistivity index (RI) of the each enlarged node were investigated.

Results: The different entiological nodes was shown a variable sonographic appearance. The Doppler indices ( $V_{\max }$ and RI) was shown nonHodgkin's lymphoma $>$ Hodgkin's lymphomas $>$ lymphoma metastases $>$ acute lymphadenitis $>$ chronic lymphadenitis $(P<0.05)$.

Conclusions: There is different echo pattern in the 91 nodes and significant difference in the Doppler indices between benign and malignant nodes, suggesting that further differentiation of malignant node may be possible. The ultrasound possesses higher clinical value.

\section{EMPHYSEMATOUS PYOMYOSITIS}

Jen-Tang Sun ${ }^{1}$, Ming-Tse Tsai ${ }^{1}$, Kuang-Chau Tsai ${ }^{1}$, Hsiu-Po Wang ${ }^{2}$, Wan-Ching Lien ${ }^{3}$

${ }^{1}$ Department of Emergency Medicine, Far Eastern Memorial Hospital, Taipei County, Taiwan

${ }^{2}$ Internal Medicine, Endoscopic Division, National Taiwan University Hospital, Department of Internal Medicine, National Taiwan University Hospital, Yunlin Branch

${ }^{3}$ Department of Emergency Medicine, College of Medicine, National Taiwan University and National Taiwan University Hospital, Taipei, Taiwan

A 46-year-old man with heroin intravenous (IV) abuser, presented with progressively left thigh pain and swelling for 5 days. He denied fever or trauma. His vital signs were normal. Physical examinations revealed diffuse left thigh tenderness without local redness and heat. Others were unremarkable. Laboratory results showed leukocytosis (23,790 per $\mu \mathrm{L})$ with left shift. Left femur radiograph and sonography showed small air pockets over the thigh, computed tomography (CT) scans revealed gas accumulation around the left thigh extending to the leg, suggestive of emphysematous pyomyositis. Our patient received antibiotics with adequate surgical drainage and he was discharged smoothly. Patient's pus culture revealed Klebsiella pneumoniae.

Pyomyositis is an uncommon disease. In temperate areas, it is associated with underlying diseases, such as diabetes and HIV infection. The quadricep muscles are the most common infection site. Symptoms include localized muscular pain, edema and lowgrade fever. The diagnosis is often delayed because it is often misdiagnosed as muscle strain in early stage, thrombophlebitis or deep vein thrombosis in later stages, perhaps, due to the lack of redness of the skin. The most common pathogen is Staphylococcus aureus. Gram negative infections are more common among patients with underlying medical illness. Klebsiella pneumoniae, is an infrequent pathogen.

Diagnosis of pyomyositis requires a high index of suspicion and can be confirmed by imaging studies. MRI is considered to be the best imaging modality for diagnosis as it shows diffuse muscle inflammation and abscess formation. The treatment of pyomyositis consists of antibiotic treatment with adequate drainage if abscess formation.

\section{APPLICATION OF THREE-DIMENSIONAL POWER DOPPLER ULTRASONOGRAPHY IN SYNOVITIS OF RHEUMATOID ARTHRITIS}

\author{
Li Shilin, Lv Guo-rong, Lin Lin, Xiao Jin-yi
}

The Second Hospital, Fu Jian Medical University, China

Objective: To investigate the value of three-dimensional power Doppler ultrasonography (3D PDU) in synovitis of rheumatoid arthritis (RA).

Methods: Wrists of 23 active and 21 inactive RA patients were examined by 3D PDU. The volume (V), vascular index (VI), PSV, EDV and RI of synoviums were measured.

Results: The V $\left(1.73 \pm 0.73 \mathrm{~cm}^{3}\right)$ and VI $(9.53 \pm 6.11)$ of active patients were higher significantly than that of inactive patients $\left(1.09 \pm 0.76 \mathrm{~cm}^{3}, P<0.01\right.$ and $\left.3.86 \pm 4.99, P<0.01\right)$, The PSV $(16.8 \pm 6.29$ vs. $13.5 \pm 8.54 \mathrm{~cm} / \mathrm{s}, P>0.05), \mathrm{EDV}(5.51 \pm 1.77$ vs. $5.03 \pm 2.76 \mathrm{~cm} / \mathrm{s}, P>0.05)$ and RI $(0.66 \pm 0.07$ vs. $0.62 \pm 0.08$, $P>0.05)$ of active patients showed no significant difference compared with that of inactive patients.

Conclusions: 3-D PDU is useful in evaluation of activity of synovitis. Measurement of VI may become a method to evaluating the activity of RA.

\section{WHAT IS THE BEST PHANTOM FOR VASCULAR ACCESS TRAINING AND CAN WE MAKE A CHEAP ONE?}

James Rippey, M. Summerscales, D. Fatovich

Sir Charles Gairdner Hospital, Dalkeith, Australia

Training people in the techniques of vascular access under ultrasound guidance should involve the use of phantoms. Training using these models allows students to gain the appropriate hand-eye coordination before attempting invasive procedures on patients. 
In this study we explore several different vascular phantoms, and through trial and error create several cheap phantoms that are compared to expensive less widely available versions.

Seven different phantoms were compared by novice sonographers completing an emergency ultrasound course. They were scored by the investigators on cost and time to be made. The sonographers evaluated the phantoms for cleanliness, reusability, realistic appearance, realistic feel on a visual analogue score chart. The scores were combined for an overall evaluation score.

The best phantom was one made from chicken, using modeling balloons as the vessels. Overall this was statistically significantly better than any other phantom. It took $3 \mathrm{~min}$ to make, cost less than AUD \$10.00, scored highest on realistic appearance and feel. It was reusable throughout the teaching session (over 20 needle passes) but obviously could not be used for more than one day. Despite being meat it scored highly on the cleanliness evaluation as it was covered in cling film and was bloodless.

The second best phantom was the AUD $\$ 750$ purchased phantom. Other phantoms tested were made from liver, silicone, and raspberry jelly. In the jelly we used psyllium husk, oatmeal and graphite particles to create fine echogenic particles. Vessels tested included balloons, latex drains and macaroni.

A 3 min video of how to create the phantom of choice will be shown in the presentation if this abstract is accepted for an oral presentation.

\section{INSPIRED-INSTRUCTION OF SONOGRAPHIC PLACEMENT OF IVS BY RNS IN THE EMERGENCY DEPARTMENT}

Andrew Liteplo, Vicki Noble, Pina Patel, Marlena Dipre, Calvin Huang, Heidi Kimberly

Division of Emergency Ultrasound, Massachusetts General Hospital, USA

Background: Ultrasound has been shown to be a valuable tool in placement of peripheral IVs, and ultrasound machines are readily available in the emergency department. We hypothesize that emergency nurses can easily learn this technique and successfully and safely use ultrasound to place peripheral IVs after a brief training course taught by emergency physicians.

Methods: Emergency physicians with prior experience with ultrasound designed a 2-h training course. Twelve emergency department nurses participated in the project. All twelve nurses completed a pre- and post-course survey of their comfort level with ultrasound. Nurses then were able to use the ultrasound to guide IV insertion in emergency patients, documenting findings and results. Patients were asked if they would prefer an ultrasound-guided IV in the future.

Results: All nurses felt comfortable with using the ultrasound machine, identifying veins, and placing IVs with ultrasound guidance after the 2-h training course. 62 patients were enrolled. Veins were rarely palpable or visible. Nurses successfully punctured a vein $86 \%$ of the time. IV access was achieved on the 1st ultrasound attempt in $56 \%$ of patients, and by the 2nd attempt in $69 \%$ of patients. Arterial puncture occurred in $1.6 \%$ of patients. Only $5 \%$ of patients would not prefer an ultrasound-guided IV in the future.

Conclusion: After a brief training session, nurses are able to use ultrasound to achieve IV access in the majority of patients, and can do so safely, accurately, and usually on the first attempt. Patients overwhelmingly would prefer ultrasound-guided IVs in the future.
DEVELOPMENT OF A NOVEL COST-EFFECTIVE, AND REPRODUCIBLE TECHNIQUE FOR MAINTAINING ASEPTIC PRECAUTIONS DURING ULTRASOUND GUIDED CENTRAL VENOUS ACCESS

Jain Anunaya, Mahesh Joshi

Department of Emergency Medicine, Apollo Health City, India

Aims: To describe development of a new cost effective technique for maintaining asepsis during ultrasound (USG) guided central venous catheterization (CVC).

Methods: A two operator technique for aseptic precautions during USG guided CVC was developed using sterile self-adhesive plastic (Tegaderm ${ }^{\circledR} 10 \times 12 \mathrm{~cm}$ ), and povidone iodine solution. To gauge coherence, 5 point Likert scale (1-strongly disagree; 5 -strongly agree) questionnaire comparing four pairs of images (1 with standard gel and 1 with new technique) was answered by 30 emergency medicine residents. Following this patients undergoing USG guided CVC with the new technique from June 2007-May 2008 were prospectively followed for 4 days. Patients $<15$ years; undergoing re-catheterization; receiving oral/intravenous antibiotics prior to procedure; with sepsis prior to $\mathrm{CVC}$; catheter removal, death or discharge within 4 days; were excluded. Post CVC infection criteria were predefined.

Results: Mean score for image coherence, brightness and contrast was 4.73 (SD 0.45), 4.9 (SD 0.62) and 4.57 (SD 0.73) respectively. All residents reported a score of 5 for anatomical identification. Of 326 patients with USG guided CVC, 186 were eligible and incidence rate for post CVC local/systemic infection was $0 \%$. 16 patients received post CVC prophylactic antibiotics. The average hospital infection rate post CVC was $7.56 \%$ over 2 years. The cost of equipment/CVC was $\$ 2.80$ compared to existing estimates of $\$ 22$ for prevalent techniques.

Conclusion: The use of Tegaderm ${ }^{\circledR}$, and povidone iodine antiseptic solution, is a cheap, reproducible, technique for maintaining asepsis during guided CVC. The adaptability of Tegaderm ${ }^{\circledR}$ to most probe types makes it potentially universally applicable for all USG guided procedures.

\section{ULTRASOUND GUIDANCE FOR INTERNAL JUGULAR CENTRAL VENOUS CANNULATION: SINGLE OPERATOR VERSUS TWO-OPERATOR VERSUS TRADITIONAL LANDMARK TECHNIQUE}

Young Soon Cho, Ki Hwan Kim, Ho Jung Kim, Hoon Kim

Soonchunhyang University Bucheon Hospital, South Korea

Background: The purpose of this study was to determine whether ultrasound guidance for internal jugular central venous cannulation by single operator and two-operator could improve success rate and decrease the number of complications compared to traditional landmark technique.

Methods: This study was a prospective, randomized, clinical trial conducted from July 2008 to September 2008 in a Korean urban teaching hospital. Patients requiring central venous access was randomized to one of the three insertion techniques (single operator technique, two-operator technique, traditional landmark technique). The primary outcome measure was cannulation success. Other outcome measure included number of attempts, access times and complication. 
Result: Thirty two patients were enrolled. Six of 7 (86\%) internal jugular vein catheters were successfully inserted by single operator technique, 13 of $14(93 \%)$ by two-operator technique and 7 of 11 $(64 \%)$ by landmark technique. First attempt cannulation was successful in 6 of 7 (86\%) using single operator technique, 9 of $14(64 \%)$ using two-operator technique and 1 of $11(9 \%)$ using landmark technique. The median start to venipuncture time was $177 \mathrm{~s}$ by single operator technique, $197 \mathrm{~s}$ by two-operator technique and $459 \mathrm{~s}$ by landmark technique. There were $6(18 \%)$ complication in the study, 5 in the landmark group and 1 in single operator group.

Conclusion: Real-time ultrasonographic-guided internal jugular vein catheterization has higher success rate, less time consuming, and lower complication rate. And single operator technique appears to be superior to the two-operator technique for first time success rate and procedure time.

\section{CAN EMERGENCY PHYSICIANS GAIN ADEQUATE CARDIAC IMAGES IN 10 SECONDS - THE "RHYTHM ANALYSIS" TIME IN ACLS?}

\author{
J. Rippey, R. Jackman, I. Jacobs
}

\section{Sir Charles Gairdner Hospital, Dalkeith, Australia}

It is very difficult to clinically exclude all the reversible causes of cardiac arrest. There is evidence that echocardiography can evaluate for tamponade, hypovolaemia, massive pulmonary embolism and can assess the degree of cardiac activity.

The aim of this study is to determine whether emergency physicians can achieve adequate subcostal cardiac views to evaluate for each of these conditions within $10 \mathrm{~s}$. We also aimed to assess whether there was a significant difference in success between emergency physician sonographers of differing expertise.

A convenience sample of 50 patients presenting to the Emergency Department consented and were examined by two emergency sonographers, one experienced and one with only $30 \mathrm{~min}$ tuition. Images were reviewed by a consultant echocardiologist who graded each view for its ability to confirm or exclude the considered diagnoses.

Overall the 10-s studies were adequate to assess for tamponade in $58 \%$, for hypovolaemia in $56 \%$, for PE in $38 \%$ and for cardiac activity in $79 \%$. The experienced emergency sonographer achieved adequate images slightly more often. There was strong correlation with adequacy of images between operators indicating that it is patient factors rather than experience that effects image quality most of the time. This small study has lead to a larger one examining integration echo into the assessment of all our arrested patients. Another exploring the amount of tuition required to interpret the images is also underway.

\section{FEASIBILITY OF POINT-OF-CARE COLOUR DOPPLER ULTRASOUND CONFIRMATION OF INTRA-OSSEOUS NEEDLE PLACEMENT DURING RESUSCITATION}

\author{
James Tsung ${ }^{1}$, Michael Blaivas ${ }^{2}$, Michael Stone ${ }^{3}$ \\ ${ }^{1}$ New York University School of Medicine, USA \\ ${ }^{2}$ Northside Hospital-Forsyth, Atlanta, USA \\ ${ }^{3}$ SUNY-Downstate College of Medicine, USA
}

Introduction: Intra-osseous needle insertion for vascular access is a standard procedure used in paediatric resuscitation. The introduction of newer automated intra-osseous devices has recently expanded its role to include resuscitation in patients of all ages. Managing resuscitation can be challenging and a misplaced intra-osseous needle may confound effective resuscitation. Colour Doppler ultrasound has been recently proposed as a method to confirm intra-osseous needle placement. The ability to rapidly determine the correct position of an intra-osseous needle during resuscitation would allow the delivery of medication or fluid infusion into the vascular space to be verified, thus optimizing resuscitation. Furthermore, complications from intraosseous infusion extravasating into soft-tissues, such as compartment syndrome, or tissue necrosis can be avoided.

Methods: We describe the point-of-care sonographic technique and colour Doppler ultrasound findings of intra-osseous needle confirmation in a case series of critically ill patients requiring resuscitation, highlighting the utility of this sonographic application.

Results: Colour Doppler ultrasound detected extra-osseous flow in incorrectly positioned intra-osseous needles, and intra-osseous flow in correctly positioned intra-osseous needles in six critically ill patients requiring resuscitation.

Conclusions: The use of point-of-care colour Doppler ultrasound to determine the location of both manually inserted or automated placement of intra-osseous access during resuscitation is feasible, can be rapidly performed, may verify delivery of resuscitative medications or infusions, and avoid complications from extravasation.

\section{USEFULNESS OF ULTRASOUND MEASUREMENT OF THE INFERIOR VENA CAVA AND AORTA DIAMETER RATIO AS AN OBJECTIVE TOOL IN THE ASSESSMENT OF DEHYDRATION IN CHILDREN}

Young-Rock Ha, Young-Sik Kim, Tae-Yong Shin, Hyun-Young Cho, Deuk-Hyun Park, Jun-Soo Kim

Dejin Medical Center, Bundang Jesaeng General Hospital, South Korea

Objectives: To verify if the IVC and Ao diameters (IVC/Ao) ratio could predict the significant dehydration in child patients, calculating the differences between the initial and last and to compare the ways of measuring the IVC/Ao ratio and figure out the best one.

Methods: This prospective observational study was performed during 7 months. Children (between 1 and 6 years of age) who were likely to be dehydrated clinically and hospitalized were enrolled. We measured the IVC/Ao ratios in longitudinal (long IVC/Ao) and transverse scan with bedside US and checked their body weight before IV hydration. In transverse scan, we measured the ratios using the long (trans-long IVC/Ao) and short (trans-short IVC/Ao) diameter of IVC. We checked body weights as the usual weight again at discharge and defined the significant dehydration as the initial weight was lost more than $5 \%$ of usual weight.

Results: 47 patients were enrolled. There are significant mean differences between dehydration group and non-dehydration group in long IVC/Ao and trans-long IVC/Ao ratios $(p=0.02$ and 0.005 , respectively). In ROC analysis, the areas under curve of long IVC/ Ao and trans-long IVC/Ao ratios to discriminate the two groups is 0.64 and 0.75 , respectively. The cut-off value of trans-long IVC/ Ao ratio was 0.876 with $80.8 \%$ of sensitivity and $60 \%$ of specificity. The classic laboratory tests such as BUN/Cr ratio, total $\mathrm{CO} 2$, and bicarbonate didn't show any differences between two groups.

Conclusions: The IVC/Ao ratio measured by bedside US is the useful tool to diagnose the significant dehydration of emergency pediatric patients. We think that could give emergency physicians important information whether the children is dehydrated or not so rapidly. It could reduce delay to hydrate and unnecessary treatment. 


\section{THE FEASIBILITY OF DIAGNOSING PAEDIATRIC} PNEUMONIA USING POINT-OF-CARE ULTRASOUND

James Tsung, Vaishali Shah, Michael Tunik

Bellevue Hospital \& New York University School of Medicine, USA

Introduction: Pneumonia (PNA) is the leading killer of children worldwide according to the World Health Organization. The WHO also estimates that up to $75 \%$ of the world's population has no access to diagnostic imaging services. Scant data exists describing the diagnosis of PNA using point-of-care ultrasound (PoCUS), and none when performed by clinicians with focused training. PoCUS diagnosis of PNA can benefit children not only in traditional healthcare settings, such as clinics and hospitals; but also in underserved areas and developing countries. We sought to determine test performance characteristics for PoCUS to diagnose PNA in children.

Methods: Prospective cohort of patients $\leq 21$ years of age presenting to an emergency department, for whom CXR was ordered to evaluate for suspected PNA. Study clinicians underwent 1 hour of focused lung US training. Informed consent was obtained from parents/ patients. Clinicians performing PoCUS were blinded to CXR results. PoCUS results were compared to radiologist reading of CXRs as reference standard.

Results: 114 children with suspected PNA were enrolled. Median age was 3 years (IQR 6), 44\% were male. The prevalence of PNA was $15 \%$. The test performance characteristics of PoCUS for PNA: sensitivity 75\% (CI 47-92\%), specificity 88\% (CI 79-93\%). Chest X-ray will miss $<1 \mathrm{~cm}$ consolidations which ultrasound can identify $\left(N^{\prime}=8\right)$. With $<1 \mathrm{~cm}$ PNAs excluded, specificity increased to $96 \%$ (CI 88-98\%).

Conclusions: Ultrasound detects $<1 \mathrm{~cm}$ PNAs that CXR can miss. It is feasible for clinicians to diagnose pediatric pneumonia using PoCUS with a high specificity rate.

\section{THE USE OF CRITICAL CARE ULTRASOUND IN THE PAEDIATRIC EMERGENCY DEPARTMENT}

\author{
Gene Yong-Kwang Ong
}

KK Women's and Children's Hospital, Singapore

Paediatric Emergency Medicine being a relatively new subspecialty is charged with the mission to quickly recognise and treat the most acute clinical conditions in the paediatric population. Point of care testing is extremely important tool. Targeted ultrasonography used in the emergency setting is goal directed and serves as an adjunct to clinical findings especially in the emergency setting. Beside targeted ultrasonography in the emergency management has been increasingly recognised as an important tool in critical management and resuscitation in most major tertiary hospitals and trauma centres.

While there have been many been many published adults studies advocating the use of targeted ultrasonography in evaluation and management in the emergency department setting in recent years, literature for its use in the paediatric population is extremely limited.

This poster illustrates the use of targeted ultrasonography in the evaluation and management of children in a Paediatric Emergency Department in a tertiary hospital in Singapore.
RECURRENT COLIC ABDOMINAL PAIN OF PERITONITIS TUBERCULOSIS AMONG THE CHILDREN (SMALL BOWEL ULTRASOUND FINDINGS)

Maria Goretti Ametembun

Maternity Emma Poeradiredja Hospital, Bandung, Indonesia

Background: This study was conducted at Maternity Emma Poeradiredja Women Hospital Bandung from 1 January 2005 to 30 April 2009 to know small bowel wall ultrasound findings of recurrent abdominal colic pain due to peritonitis tuberculosis among the children.

Methods: The children with disseminated tuberculosis (lung, cervical lymphadenopathy and tuberculous dry type peritonitis) diagnosed based on clinically, laboratory, thorax photo, PPD test and ultrasound. The recurrent colic abdominal pain and tenderness were associated with the doughy abdomen and dullness percussion area of dam-board phenomena of tuberculous peritonitis. Small bowel wall thickness examined with transabdominal ALOKA Prosound SSD3500 on the tympanic area and dullness area according of dam-board phenomena. Results: 82 children of $2.5-13$ years, mean 6.2 years, $44(53.66 \%)$ male, $38(46.34 \%)$ female. The mean of small bowel wall thickness on the tympanic area was $0.16 \mathrm{~cm}$ otherwise on dullness area was $0.42 \mathrm{~cm}(2.62$ times than tympanic area). Hypoperistaltic movement, loss differentiation of the wall layers, irregular margin of the small bowel wall, and irregular oval/round lesions (patchy echogenic nonshadowing with an irregular rim of lower echodensity) within the affected area.

Conclusion: Small bowel wall thickening, hypoperistaltic movement, irregular margin and oval/round lesions (patchy echogenic nonshadowing with an irregular rim of lower echodensity) within the wall were found on affected dullness area of peritonitis tuberculosis among the children.

\section{BEDSIDE ULTRASOUND PERFORMED BY EMERGENCY RESIDENT PHYSICIANS CAN PROVIDE EARLY AND ACCURATE DIAGNOSIS FOR PAEDIATRIC INTUSSUSCEPTION IN THE EMERGENCY DEPARTMENT}

\author{
Kuo-Chih Chen, Liang-Ting Wu, Chee-Fah Chong, \\ Tzong-Luen Wang
}

Emergency Department, Shin-Kong Wu Ho-Su Memorial Hospital, Taiwan

Objectives: Early diagnosis of paediatric intussusception can facilitate prompt treatments and prevent complications. The aim of this study was to investigate whether bedside ultrasound performed by emergency physicians can provide early and accurate diagnosis for paediatric intussusceptions.

Methods: We retrospectively reviewed 28 medical records from patients younger than 18 years old with a diagnosis of intussusceptions in the emergency department from 1 January 2002 to 31 March 2009. All of the ultrasound scans were performed by the emergency physicians and then confirmed by one senior paediatric gastrointestinal specialist.

Results: Among these 28 patients, there were 19 boys and 9 girls, aged from 10 months to 7 years. The major causes for emergency department visiting were abdominal pain $(71.4 \%)$, vomiting $(42.9 \%)$, fever $(14.3 \%)$, irritable crying $(10.7 \%)$, and bloody stool $(7.1 \%)$. Three $(10.7 \%)$ patients were diagnosed by first year emergency residents, $20(71.4 \%)$ by second year emergency residents, and $4(14.3 \%)$ by third year emergency residents. The attending emergency physicians 
diagnosed one (3.6\%) patient and supervised all scans performed by emergency residents. The median and mean time from emergency department registration to bedside ultrasound diagnosis were 29 and $58 \mathrm{~min}$. The median and mean time from registration to formal pediatric ultrasound diagnosis were 184 and $289 \mathrm{~min}$. Twenty five $(89.3 \%)$ patients had successful hydrostatic enema reduction, two $(7.1 \%)$ had spontaneous reduction, and one (3.6\%) had surgical reduction.

Conclusions: The present study shows that emergency resident physicians can promptly and accurately diagnose paediatric intussusceptions by bedside ultrasound in the emergency department.

\section{VALIDATION OF NURSE PERFORMED FAST ULTRASOUND}

Justin Bowra ${ }^{1}$, Sally Horder ${ }^{2}$, Erica Caldwell ${ }^{2}$, Michelle Cox ${ }^{2}$, Scott D'Amours ${ }^{2}$

${ }^{1}$ Sydney Adventist \& St Vincent's Hospitals, Australia

${ }^{2}$ Liverpool Hospital, Sydney, Australia

Background: Patients presenting to Emergency Departments (EDs) with abdominal trauma benefit from FAST (Focused Assessment with Sonography in Trauma). Not all doctor members of the trauma team are credentialed in FAST; therefore occasionally no one is available in the hospital to undertake a FAST. Hence, the aim of this study was to determine the accuracy of nurse-performed FAST as a practical alternative where suitably trained doctors are not available.

Methods: This was a prospective study of a convenience sample of patients with multisystem trauma in whom abdominal injury was clinically suspected. Senior nurses trained in FAST performed and reported FAST scans for each patient. Accuracy of nurse-performed FAST was determined by comparing results with Computerised Tomography (CT) scan, laparotomy or clinical course.

Results: 552 indicated nurse-performed FAST scans were included in the study. Nurse-performed FAST demonstrated sensitivity of $85.2 \%$ (95\% CI 73.3-92.6) and specificity of 99.4\% (95\% CI 98.1-99.8), a positive predictive value (PPV) of $94.5 \%$ (95\% CI 83.9-98.6) and a negative predictive value (NPV) of $98.2 \%$ (95\% CI 96.5-99.1). Overall accuracy of nurse-performed FAST for the detection of free fluid was $97.8 \%$ (95\% CI 96.1-98.8).

Conclusion: This study demonstrates that, in a convenience sample of injured patients, nurse-performed FAST achieved similar accuracy to previously published results of doctor-performed FAST. Future studies with greater patient numbers would be valuable.

\section{IS THERE A LINK BETWEEN "LUNG ROCKETS" SIGN AND HIGH NTPRO-BNP LEVELS IN PATIENTS WITH CONGESTIVE HEART FAILURE IN PRE-HOSPITAL SETTING?}

\section{Gregor Prosen, Stefek Grmec}

Centre for Emergency Medicine, Community Health Centre Maribor, Slovenia

Introduction: "Lung rockets" artefacts on chest US is believed to represent alveolar or interstitial lung edema. Similarly, high NTproBNP levels point to heart failure with high specificity.

Methods: This was retrospective observational study in pre-hospital setting. We performed chest US in 30 patients that presented with signs and symptoms indicative of heart failure. We looked for lung rockets in anterior and lateral chest areas and categorized an exam as "lung rockets present" in case we could show them bilaterally. In all patients with lung rockets present, we measured serum levels of NTpro-BNP. All patients had pulmonary edema confirmed radiographically subsequently.

Results: We measured NTpro-BNP levels in 30 patient with lung rockets present bilaterally in anterior chest region and 23 of them (77\%) had levels higher then $3,000 \mathrm{pg} / \mathrm{ml}$ (t.i. unmeasurable exact level). Other $7(23 \%)$ had values between 1,250 and 3,000 $(1,753 \pm 383 \mathrm{pg} / \mathrm{ml} ; 95 \%$ CI: $1,398-2,108)$.

Conclusion: All patients with signs and symptoms of left-sided heart failure and lung rockets sign present bilaterally, had levels of NTproBNP severely elevated, all of them over the values that are set to indicate heart failure with great certainty. This preliminary results form a base for prospective study evaluating use of rockets sign and NTproBNP levels in dyspnoic patients, to differentiate between CHF and COPD/asthma as cause of acute dyspnea.

\section{ULTRASONOGRAPHIC APPLICATION IN RESCUING VICTIMS CAUSED BY WENCHUAN EARTHQUAKE}

\author{
Deng Dan, Tan Yan
}

Department of Ultrasound, General Hospital of Chengdu Military Command, China

Objective: To investigate the role of ultrasonography in rescuing the victims caused by Wenchuan earthquake.

Methods: We analyze retrospectively the ultrasonography application range and methods as well as the role of ultrasonograph in rescuing the earthquake victims.

Results: 1,207 of 3,207 wounded patients (37.64\%) received ultrasound examination. A follow-up of ultrasound was performed in some patients, and the number of ultrasonograph reached 1,386 in total. $6.96 \%$ (115/ 1386) patients received ultrasound-guided interventional treatments. Hemoperitoneum was found in 16 cases by ultrasound screening. In addition, 45 cases of hemothorax, 1 case of arterio-venous fistula following trauma, 3 cases of deep veins phlebothrombosis of lower limb following fracture and 12 cases of deep part hematoma of soft tissue were detected by ultrasonograph. Meanwhile, we followed up the changes of renal volume, renal blood flow as well as serous effusions in the process of treatments of crush syndrome. Visceral injury was confirmed by surgery or CT later in 5 cases of negative seroperitoneum by ultrasonograph. All the ultrasound-guided interventional treatments were performed successfully. Trauma-associated pathology was detected in 6.96\% (84/ 1207) of the patients by ultrasound screening with $0.45 \%(5 / 1123)$ false negatives and no false positives. The number of bedside ultrasonography was $73.87 \%$ (458/620) of ultrasound examinations in the middle and later stage (72 h-4 w after earthquake).

Conclusions: Ultrasonography was widely used not only in triage of earthquake victims, interventional treatments, but also to perform bedside examination for severe cases, which play a great role in rescue tasks after earthquake.

\section{ESTABLISHING PRE-HOSPITAL AND RETRIEVAL ULTRASONOGRAPHY FOR ADULT HEMS RETRIEVAL SERVICES IN NEW SOUTH WALES}

\section{F. H. Cummins, C. Reid}

Careflight, Westmead Hospital, Australia

Aim: To describe the process by which handheld ultrasonography has been introduced into adult Helicopter Emergency Medical Service (HEMS) in New South Wales. 
Methods: A review of the implementation process was undertaken. Results: The introduction of handheld ultrasonography to the adult retrieval services in New South Wales followed a systematic approach. Initially clinicians' opinions were sought via a detailed questionnaire in order to identify issues for policy development and training prior to implementation of handheld ultrasonography. Results were summarised and shared with the physician team. Following this a dedicated training course addressed specific training needs for the pre hospital and retrieval environments. Concurrently an operational policy was constructed to address governance issues including audit, training and ongoing verification.

Conclusion: The structured approach employed in the introduction of handheld ultrasonography addressed specific needs for the pre hospital and retrieval clinicians.

\section{PARAMEDIC PERFORMED THORACIC ULTRASOUND FOR PNEUMOTHORAX DIAGNOSIS}

\author{
Vicki Noble ${ }^{1}$, Andrew Liteplo ${ }^{1}$, Alice Murray ${ }^{2}$ \\ ${ }^{1}$ Division of Emergency Ultrasound, Massachusetts General Hospital, \\ USA \\ ${ }^{2}$ Royal Infirmary of Edinburgh, UK
}

Background: Previous research has shown that thoracic ultrasound is an accurate way to assess for pneumothorax and that novice users perform well on post training image interpretation testing. We evaluated the accuracy of thoracic ultrasound performed by trained medics during transport without expert image acquisition or interpretation supervision. Methods: Paramedics completed a thoracic ultrasound training module. Post training, a Siemens P10 (2-5 MHz phased array probe) or a Sonosite 180 (5-12 MHz linear probe) was used to perform thoracic scans on transported patients, recording real-time interpretations. Saved clips were reviewed by an ultrasound fellowship trained emergency physician and compared to gold standard imaging diagnostics at the receiving hospital. Results: Over a two month period, 35 scans were logged. 25 scans were performed on the Siemens P10 and 10 scans were performed on the Sonosite 180. Of the 35 scans performed, 14/35 had adequate images to support the image interpretation on review. The paramedics were able to interpret $7 / 7$ of the linear probe acquired and 23/28 of the phased array acquired images. The accuracy of the image interpretation was $26 / 35 \mathrm{TN}, 4 / 35 \mathrm{FN}$, and 5/35 the medic could not interpret secondary to poor views. These numbers give a negative predictive value of 0.87 and indicate a feasibility of $86 \%$.

Conclusions: While the negative predictive value of paramedic performed thoracic ultrasound is lower than that previously reported, it is still sufficiently high to be clinically useful. However, it appears that paramedics have more difficulty calling a scan positive in clinical situations.

\section{IMPLEMENTING HANDHELD ULTRASONOGRAPHY IN PRE-HOSPITAL AND RETRIEVAL MEDICINE - FROM CONSULTATION TO PRACTICE}

\author{
Karel Habig ${ }^{1}$, Cliff Reid $^{1}$, Fergal Cummins ${ }^{2}$ \\ ${ }^{1}$ Ambulance Service of New South Wales, Australia \\ ${ }^{2}$ CareFlight, Westmead, Sydney, Australia
}

Aim: To describe the implementation process for the introduction of handheld ultrasonography into a Helicopter Emergency Medical Service (HEMS).
Results: A four stage process was followed.

Stage one: a confidential questionnaire was emailed to all physicians to quantify ultrasonographic experience and any concerns over the use of ultrasonography in HEMS missions. Results were summarised and shared with the physician team.

Stage two: a dedicated retrieval ultrasound training day to address learning needs and concerns identified in stage one was held, combining didactic lectures with practice on human volunteers and phantoms.

Stage three: a standard operating procedure was introduced defining role and process for retrieval ultrasonography.

Stage four: a dedicated audit pro-forma was designed to accompany the introduction of the ultrasound devices into operational use. This enabled standard reporting documentation in addition to continual review of standard operating procedure adherence and real time feedback of operator experience.

Discussion: Pre- and inter-hospital ultrasonography use lacks a strong evidence base and therefore continual monitoring is required to ensure appropriateness of application. Concerns by physicians over its use may be addressed both by encouraging ongoing feedback mechanisms and by the provision of structured training.

Conclusion: Our four-stage approach attempted to address physician concerns, training needs, and clinical governance requirements. It may serve as model for the introduction of new technologies or procedures into a critical care service.

\section{THE DEVELOPMENT OF A DEDICATED PRE-HOSPITAL AND RETRIEVAL ULTRASOUND COURSE FOR HEMS RETRIEVAL SERVICES}

\author{
Karel Habig ${ }^{1}$, Cliff Reid ${ }^{1}$, Fergal Cummins ${ }^{2}$, Justin Bowra ${ }^{3}$, \\ Tony Joseph ${ }^{4}$, Louisa Chan ${ }^{5}$ \\ ${ }^{1}$ Ambulance Service of New South Wales, Australia \\ ${ }^{2}$ CareFlight, Westmead, Sydney, Australia \\ ${ }^{3}$ St. Vincent's Hospital, Sydney, Australia \\ ${ }^{4}$ Royal North Shore Hospital, Sydney, Australia \\ ${ }^{5}$ Basingstoke Hospital, Basingstoke, UK
}

Aim: To describe the process involved in the development of an ultrasound course with a specific emphasis on the requirements of adult HEMS retrieval services in New South Wales.

Methods: An inclusive approach was utilised optimising the expertise of local retrieval services and international experts. Prior to the course a confidential questionnaire was circulated to retrieval physicians focusing on their expectations, needs and concerns for utilising ultrasound in the pre hospital and retrieval environments. A standard ultrasound course model was used as an initial template. This template was added to with a focus on the findings of the initial questionnaire, specific encountered clinical conditions, dedicated portable equipment, the pre hospital and inter hospital environments and retrieval personnel. The format of the course included didactic teaching, small group learning and hands-on learning components. Course assessment and critique are further described.

Conclusion: The focused approach employed in the development of this course addressed specific needs and utilised the local experience of experts in the fields of HEMS pre hospital retrieval and ultrasonography. This approach may assist in the development of other such courses. 


\section{INTRODUCING HANDHELD ULTRASONOGRAPHY TO A HEMS PRE-HOSPITAL AND RETRIEVAL MEDICINE SERVICE: CANVASSING PHYSICIANS' CONCERNS}

\author{
Karel Habig ${ }^{1}$, Cliff Reid ${ }^{1}$, Fergal Cummins ${ }^{2}$ \\ ${ }^{1}$ Ambulance Service of New South Wales, Australia \\ ${ }^{2}$ CareFlight, Westmead, Sydney, Australia
}

\begin{abstract}
Aim: To identify issues for policy development and training prior to implementation of handheld ultrasonography into a Helicopter Emergency Medical Service (HEMS).

Methods: A confidential email questionnaire survey sent to all HEMS physicians to quantify prior ultrasonography training and accreditation and to invite comments on the role of ultrasonography in prehospital and retrieval medicine. Results were summarised and fed back to the group.
\end{abstract}

Results: 33 of $53(62 \%)$ physicians responded. 28/33 regularly used ultrasound in their hospital practice, although 18 had not undergone any formal accreditation process. Vascular access was the commonest application which respondents were confident to undertake unsupervised, followed by FAST, abdominal aorta, femoral nerve block, basic echocardiography, and pneumothorax assessment.

For inter-hospital missions, vascular access and haemodynamic assessment were felt to be the most useful applications. For prehospital missions, pneumothorax detection was felt to be the most useful. Several respondents expressed concern that pre-hospital FAST may contribute to delays without influencing triage decisions or prehospital therapy. Concerns were also expressed regarding quality assurance of ultrasound use and image interpretation.

Conclusion: A confidential survey highlighted educational needs and physician concerns regarding the use of ultrasonography in our HEMS. This provided essential information from which to plan subsequent training and operational policy.

\section{REAL-TIME WIRELESS VIDEO STREAMING OF ULTRASOUND TO AN IPHONE}

\author{
Andrew Liteplo ${ }^{1}$, Vicki Noble ${ }^{1}$, Ben Attwood ${ }^{2}$ \\ ${ }^{1}$ Division of Emergency Ultrasound, Massachusetts General Hospital, \\ USA \\ ${ }^{2}$ University of Oxford, John Radcliffe Hospital, UK
}

Background: Remote transmission of real-time ultrasound has great potential as a diagnostic tool in pre-hospital and rural environments. Recent innovations in technology have made live video-streaming to a handheld cell phone over a wireless network possible. We hypothesized that such technology could be used to streamline live video of ultrasound with speed and clarity sufficiently high enough to make real-time assessments of ultrasound images remotely.

Methods: An ultrasound machine was connected via an S-video cable to an audio digital video converter which connected via a Firewire cable to a laptop. The laptop, which connected to the internet via a wifi network, streamlined the video and audio input through a free software download. A handheld phone with an inexpensive downloaded application received the images via a $3 \mathrm{G}$ network connection. The transmission was done across the Atlantic Ocean, from Boston, USA, to Oxford, UK. The sending physician scanned random video clips typical to emergency ultrasound in real-time. The sending and receiving physicians communicated via a free internet-based telephone.
Results: The delay in transmission was $2-3$ s. The receiving physician was able to accurately identify the part of the body and orientation $100 \%$ of the time. The image clarity was always good enough to identify the presence or absence of pathology.

Conclusions: Real-time transmission of ultrasound video to an iPhone is feasible, and can be done so rapidly and with good preservation of image quality. This technology has the potential to be very useful in remote and pre-hospital settings. Further studies are needed.

\section{THE PRE-HOSPITAL ULTRASOUND STUDY}

David Hildebrandt, William Heegaard, Jeff Ho, Scott Joing, Robert Reardon

Hennepin County Medical Center, Minneapolis, USA

Background: Pre-hospital US has not yet been evaluated in the United States, but has been shown to be useful in Europe. We present initial study results of our pre-hospital paramedic (PM) US study.

Objective: Our Pre-hospital PM US study will train 911 PM's to be competent in using point of care US for the FAST (Focused Assessment Sonography in Trauma) and abdominal aorta (AA) exams.

Methods: This was a prospective observational study on the use of US in the pre-hospital setting. PMs were trained through lecture, written pre/ post exams and Objective Structured Clinical Examination (OSCE) format in a six hour training program. Sonosite Micromaxx US machines with a P17 probe were used. All US views were recorded using six second video clips. These studies were then over-read by an independent, Emergency Ultrasound Fellowship Director.

Results: One hundred four patients had an US performed during convenience sampling between 1 January 2008, and 1 January 2009. Twenty AA exams were performed and all were interpreted as negative by the PMs and the physician over reader (PO). Eighty four patients had the FAST exam performed. PMs were unable to obtain adequate images in $9.5 \%(8 / 84)$ of the patients. Six exams $(6 / 84$, $7.1 \%$ ) were read as positive for free intra-peritoneal fluid by both the PM and PO. FAST exam interpretation by the PM's had a sensitivity and specificity of $100 \%$ using the PO's interpretation as the gold standard.

Conclusion: With close supervision paramedics can obtain and interpret pre-hospital FAST and AA US images with a high degree of accuracy.

\section{CAN PARAMEDICS USE PORTABLE ULTRASOUND TO IDENTIFY FREE ABDOMINAL FLUID AND ABDOMINAL AORTIC ANEURYSM FOLLOWING A SHORT COURSE?}

Jim Connolly, Tricia Scott, Iain Nellis, Julia Williams, Malcolm Woollard

Method: A convenience sample of 9 paramedics completed a training programme comprising 1-day theory and 2-days supervised practice on known renal and AAA patients. Following 6-months the paramedics were assessed using portable ultrasound on 30 patients, 10 of whom had abdominal fluid, 10 who had AAA and 10 who had neither of these conditions. Patients were randomised and diagnosis was unknown to the paramedics who were assessed by a certificated examiner using Objective Structured Clinical Examination (OSCE) conditions.

Results: Paramedics conducted 78 clinical assessments and results suggest accumulative competence with practice concerning use of controls, orientation, range of views and quality of the image. Data 
will be presented to show the feasibility of such training and the areas where skill acquisition is easiest. A standard method for diagnostic testing was used comprising sensitivity, specificity, negative and positive predictive value, and accuracy to determine the value of the didactic and hands-on training programme.

Conclusions: There is potential, following adequate training, for UK emergency paramedics to use prehospital portable sonography for specific abdominal conditions. However, there is currently no ultrasound practice competency for Emergency Paramedics on the UK Health Professionals Council Register. Educational preparation of emergency paramedics in the use of portable ultrasound to identify free peritoneal fluid and AAA requires sufficient theoretical and practice preparation with implications for fitness to practice. A potential model for care will be discussed.

\section{COMPARATIVE ANALYSIS OF MYOCARDIAL CONTRAST ECHOCARDIOGRAPHY AND THE GRADE OF TIMI OF CORONARY ARTERY}

\author{
Mu Yu-ming, Li Feng-wu Qi, XiaYang, Zhi-yingyuan
}

Department of Echo-cardiograph at the First Affliated Hospital of Xinjiang Medical University, China

Objective: To investigate the diagnostic value of myocardial contrast echocardiography (MCE) and the grade of TIMI in evaluating myocardial microcirculation perfusion and clinical applications. To assess myocardial perfusion by MCE in patients with coronary artery disease before and after PCI surgery.

Methods: Thirty AMI and eleven ACS patients were examined by MCE using Sonovue intravenous injection before selective coronary angiograph. Thirty-six of patients were examined by MCE again after PCI surgery. The images of MCE were analyzed quantitatively from microbubble replenishment curves for myocardial perfusion. Left ventricular myocardial segments, divided into 4 groups according to the grade of TIMI of coronary artery detected by coronary artery angiography. Acoustic density parameters were compared among these groups, and the correlation between these parameters and the grade of TIMI of coronary artery. Results: Results among the four groups were significantly respectively $(P<0.05)$, the A, $\beta$ and A. $\beta$ were decreased significantly with the grade of TIMI of coronary artery (all $P<0.05$ ); Before PCI surgery, A, $\beta$, and A. $\beta$ were significantly lower in the regions of interest with severe lower the grade of TIMI of coronary artery than those with normal coronary artery $(P<0.05)$. The normalised value of $\mathrm{Ar}, \beta \mathrm{r}$, and $\mathrm{A} \cdot \beta \mathrm{r}$ were higher after PCI surgery than those before operation $(P<0.05)$.

Conclusion: Perfusion abnormalities are present in patients with severe lower the grade of TIMI of coronary artery and can be detected by MCE. However, myocardial microcirculation perfusion does not correlate with the grade of TIMI of coronary artery completely. Furthermore, MCE can provide valuable information on the outcome of coronary artery PCI surgery in these patients.

\section{FEATURE OF CONTRAST ENHANCED ULTRASOUND IN CLEAR CELL RENAL CARINOMA: CYSTIC, HEMORRHAGIC, DEBRIS AND NECROTIC TISSUE AND PSEUDOCAPULE}

Bin Yang

Ultrasound Association, China

Objective: To investigate present rate of a pseudocapule and cystic, hemorrhagic, debris and necrotic tissue in clear cell renal cell carcinomas (RCCs) on contrast- enhanced sonography compared with conventional gray-scale sonography.

Methods: 184 patients with RCCs proved by histologic diagnosis were prospectively studied with contrast-enhanced sonography compared with conventional gray-scale imaging. The present rate of a pseudocapule and cystic, hemorrhagic, debris and necrotic tissue in RCCs was assessed on contrast- enhanced sonography compared with conventional gray-scale sonography.

Results: On conventional sonography, the presence of a pseudocapsule was detected in 63 of 184 RCCs. Sonographic contrast-enhanced imaging revealed the in 87 of 184 RCCs presence of pseudocapsule in 107 of 184 RCCs $(P<0.05)$. On conventional sonography, the presence of cystic, hemorrhagic, debris and necrotic tissue were seen in 87 of 184 RCCs. The cystic, hemorrhagic, debris and necrotic tissue were visible in 134 of 184 RCCs.

Conclusions: Sonographic contrast-enhanced imaging is effective in improving the sonographic visualization of pseudocapsule and cystic, hemorrhagic, debris and necrotic tissue in RCCs. Ultrasonic diagnosis could supply more information in RCCs.

\section{COMBINED HAEMOSTATIC PERCUTANEOUS INJECTION THERAPY FOR SEVERE HEPATIC TRAUMA GUIDED BY CONTRAST-ENHANCED ULTRASONOGRAPHY}

\section{Zhiyan Li, Jie Tang, Faqin Lv, Yukun Luo, Tengfei Yu,} Qin Song, Ziyu Jiao

Department of Ultrasound, Chinese People's Liberation Army General Hospital

Objective: To explore the value of contrast-enhanced ultrasound (CEUS) guided percutaneous injection therapy for grade III-IV hepatic trauma patients.

Material and Methods: Fourteen patients with severe hepatic trauma were treated using the percutaneous injection of hemostatic agents. Six of them were hepatic injury of grade III, and others were grade IV according to AAST scale. Immediately after the procedure and at 3 days, and 1 and 2 weeks, and 1 and 3 and 6 months after procedure, CEUS or CT was performed in 12 patients.

Results: Out of all patients with severe hepatic trauma, 12 patients were successful once. Rehemorrhage occured in 1 patient, who received second injection therapy. One patient was switched to surgery because of concomitant pancreatic injury. During the follow up biliary fistula occurred in one patient who recovered completely by drainage. The hepatic injuries sites became deflated to disappear gradually.

Conclusions: Combining advantage of CEUS in diagnosis of bleeding of the hepatic trauma, with the technology of interventional ultrasonography, the method of CEUS guided percutaneous injection therapy for hepatic trauma with grades III-IV can save life and avoid resecting organ, and can save time for treatment of other injury companied. Therefore, it will be potential for wide application.

\section{EVALUATION AND APPLICATION OF VASCULAR ENHANCEMENT TECHNOLOGY: A NEW METHOD OF ULTRASONIC IMAGING}

Yun You Duan, Xi Liu, Jia Wang, Juan Li, Wei Hua Hou, Tie Sheng Cao

Department of Ultrasound Diagnostics, Tangdu Hospital, Fourth Military Medical University, China 
Purpose: Vascular enhancement technology (VET) is a new form of ultrasonographic technology which can optimize the image by enhancing the B-mode display with information of blood flow. The purpose of this study was to design an in vitro model to evaluate the accuracy and application method of VET, and to apply this technology preliminarily in vivo in the vascular periphery.

Method: An in vitro model was designed with a flow pump system to simulate blood flow of human vasculature. Modeling vessels were imaged by traditional B mode, color Doppler imaging and VET. The diameter of the various silicon tubes was measured to verify the accuracy of VET. For in vivo application, 15 normal subjects and 26 patients suspected of cerebrovascular disease were examined using three image modes. The imaging effects were observed and compared.

Results: VET imaging was able to clarify the lumens of the modeling vessels and reduce artifacts. Caliber of three different sizes of silicon tubing was also measured accurately by VET. Of 15 normal subjects, sound artifacts in large vessels obviously were inhibited and the inter-media membrane was displayed clearly by VET. The boundaries of carotid plaque were manifested by VET with well-defined edges. Three cases of hypoechoic soft plaque on the anterior wall that were missed in B-mode imaging were detected by VET study. Intracranial scanning with VET identified cerebral vascular disease including cerebral stenosis, arteriovenous, and aneurysm.

Conclusion: VET is helpful in improving the boundary detection of vessels and the visualization of micro-vasculature.

\section{ULTRASOUND USE IN CONDUCTED ELECTRICAL WEAPON RESEARCH (extended abstract)}

Robert Reardon ${ }^{1}$, James Miner ${ }^{1}$, William Heegaard ${ }^{1}$, Donald Dawes ${ }^{2}$, Jeffrey $\mathrm{Ho}^{3}$

\author{
${ }^{1}$ Department Emergency Medicine, Hennepin County Medical \\ Center, USA \\ ${ }^{2}$ Department of Physiology and Biophysics, University of Louisville, \\ USA and Santa Barbara Police Department, USA \\ ${ }^{3}$ Department Emergency Medicine, Hennepin County Medical \\ Center, USA and Meeker County Sheriff's Office, Litchfield, USA
}

Introduction: The TASER Conducted Electrical Weapon (CEW, TASER International, Scottsdale, AZ) is a law enforcement tool that causes neuromuscular incapacitation in violent suspects. CEW use is gaining popularity worldwide. The CEW is often used on suspects that require emergency medical attention following the law enforcement interaction. This may be due to factors such as behavioral control problems, metabolic acidosis, or drug intoxication. Occasionally, death has occurred temporally following CEW use. CEWs utilize electrical current for effect. The relationship between CEW use and death has been hypothesized to be via direct inducement of cardiac arrhythmia. Artifact from CEW application prevents real-time monitoring of research subjects using traditional means such as cardiac monitors. This project describes the novel use of ultrasound as a research tool that successfully allows real-time CEW physiology evaluation in this emerging area of research. This research should be of interest to field paramedics, emergency physicians, critical care clinicians, cardiologists and medical examiners because they are often called upon to evaluate or manage patients who have experienced sudden death after law enforcement interaction. They are also called upon to provide opinions about the mechanisms of death and there have been several speculative opinions proffered by this population that have been directly refuted by human research evidence. Ultrasound use has allowed more accurate conclusions to be made regarding these opinions. We present the first known comprehensive description of this ultrasound application in the CEW research environment. This abstract demonstrates the utility and contribution of ultrasound technology in aiding the scientific understanding of how CEWs affect human physiology.

Methods: After informed consent, volunteers have participated in a number of CEW studies. These studies have examined various CEW models as well as application locations, durations and subject conditions. Before, during and after CEW exposure, cardiac and diaphragmatic sonography with a SonoSite M-Turbo (SonoSite, Inc., Bothell, WA) is performed with standard windows. Real-time 2D imaging allows direct observation of diaphragmatic excursion (indicating respiration) and gross cardiac activity during CEW exposure. M-mode imaging of the mitral valve or left ventricle can be used to determine the cardiac rate and rhythm. Data is compiled in a database for analysis.

Results: Successful sonographic imaging of the heart and/or diaphragm has occurred in 113 subjects during real-time CEW exposure. Sonography has allowed us to definitively determine whether CEW exposure causes "capture" of the heart or diaphragm. Some of these findings have been published in several original research studies or reports

Conclusion: Ultrasound is effective for directly observing the effects of CEWs on human organs in real-time. No other monitoring or imaging modality can provide this valuable information. We present several examples to demonstrate the utility of ultrasound in CEW research. Sonography should continue to be part of the CEW comprehensive evaluation process and this non-traditional application of ultrasound technology will continue to evolve.

\section{SOLAR-POWERED ULTRASOUND RESEARCH EXPEDITION ON MT. KILIMANJARO (19,340 FT)}

\section{N. Stuart Harris} Department of Emergency Medicine, Massachusetts General
Hospital. Harvard Medical, USA

We report on an 'off-the-grid', solar-powered ultrasound research expedition on Mt. Kilimanjaro (19,340 ft) in 2009. The high altitude, remote nature of our research required that all equipment be hand-carried and use portable, 'off-the-grid' electrical power. After evaluating all currently available options, for reasons of cost, weight, portability, and reliability, we chose electrical current provided by photovoltaic solar arrays. Our primary electrical draws were our ultrasound unit (Sonosite 180) and our solid state laptop computer (Dell Inspiron 910). To power these devices, we channelled two $32 \mathrm{w}$ rigid solar arrays through an electronic voltage control device linked to a 16 Amp-Hour lead acid battery storage system (available through CTSolar.com). Fully charged, our battery system provided approximately $3 \mathrm{~h}$ of active scanning time. Obviously, during daylight hours, when solar power may continue to be generated, this time could be significantly longer. The ultimate output of any solar array is influenced by length of day, latitude, season, and cloud cover. Difficulties experienced included the lack of 'direct' low-voltage DC current capability of our Sonosite 180which required that battery current be channelled first through a $12 \mathrm{~V} \mathrm{DC}$ to $120 \mathrm{~V} \mathrm{AC}$ 'step up' converter, then through a $120 \mathrm{~V}$ AC to $16 \mathrm{~V}$ DC 'step-down' converter (as would be used in a standard in-hospital setting). These conversions introduced significant and needless power loss. Despite investigations, no currently available system obviates this requirement. We believe the technical difficulties overcome by our team will be educational to future ultrasound research expeditions. 


\section{ACQUISITION OF ULTRASOUND IMAGES OVER THE INTERNET IN REALTIME USING FREE-TO-DOWNLOAD VIDEOCONFERENCING SOFTWARE}

\author{
Ben Attwood ${ }^{1}$, Andrew Liteplo ${ }^{2}$ \\ ${ }^{1}$ Nuffield Department of Anaesthetics, Oxford, UK \\ ${ }^{2}$ Vicki Noble, Department of Emergency Medicine, Massachusetts \\ General Hospital, USA
}

Background: With the advent of affordable computers capable of transmitting video images and sound through videoconferencing software and mobile internet capabilities, it may be possible to use this technology to transmit ultrasound data for clinical and education use.

Objective: A proof-of-concept trial to establish the feasibility and technical capabilities of a novel method of transmitting ultrasound video and still images transmitted over the internet, viewed and saved in real-time using videoconferencing software and inexpensive computing hardware.

Methods: The real-time output display of a portable battery-powered ultrasound machine was captured by a laptop computer via an analogue to digital converter. The captured display was then sent in real-time using two free-to-download videoconferencing software programs to a recipient on another continent (USA to UK). Both videoconferencing programs sent data via two methods of internet access, domestic broadband (WiFi) and third generation mobile broadband (3G). Still images and videos were recorded on the recipient laptop and subsequently evaluated for quality, detail and resolution by three hospital emergency sonologists. Image quality was compared against speed of data transmission (bandwidth).

Results: In most cases image quality, resolution and detail was of high quality. There was good interrater agreement between the reviewers. There was good correlation between bandwidth and data quality. Below a threshold bandwidth $(<70 \mathrm{kbp})$ images were not interpretable.

Conclusion: Ultrasound images can be transmitted using conventional and inexpensive equipment. Providing sufficient bandwidth, the quality, detail and resolution is sufficient to use in clinical scenarios such as in the pre-hospital environment. More studies are needed.

\section{IS TRADITIONAL ULTRASOUND TRAINING THE RIGHT WAY TO TEACH POINT OF CARE CLINICIANS, OR DO THEY REQUIRE PERSONALISED EDUCATION?}

\author{
S.P. Richards ${ }^{1}$, J. Connolly ${ }^{2}$, R.D. Jarman ${ }^{3}$ \\ ${ }^{1}$ Teesside University, Middlesbrough, Tees Valley, UK \\ ${ }^{2}$ Newcastle General Hospital, Newcastle, Tyne and Wear, UK \\ ${ }^{3}$ Queen Elizabeth Hospital, Gateshead, Tyne and Wear, UK
}

Introduction: As point-of-care (POC) ultrasound developed within the UK, a need was identified for the delivery of quality ultrasound training, to include techniques, governance, equipment and safety. In 2004, Teesside University, started to deliver this training in focused courses.

Discussion: Over a 5 year period the learning, teaching and assessment strategies of the courses were reviewed and feedback obtained from alumni and their clinical mentors/trainers, and enhancements made to the courses. Initially the courses were delivered within an existing, ultrasound programme. Learning was delivered with sonography students over blocks and teaching methods included lectures, group work and problem solving exercises. Students were expected to complete a set number of practice hours at a clinical placement and had a mentor to assist them in making the theory practice link. Whilst this style of delivery suits student sonographers, it proved problematic to POC clinicians and issues with retention and completion were identified. Over time, the duration of the courses were extended, allowing clinicians to gain the necessary competence; the assessment strategy was changed from a portfolio to written exam and the delivery altered, incorporating more intense, concentrated sessions and practical workshops. The areas in which POC ultrasound differed from traditional ultrasound examinations were addressed and dedicated sessions included. These enhancements improved feedback, retention and completion enabling clinicians' access to quality ultrasound education.

Conclusion: Designing personalised ultrasound courses for point of care clinicians gives greater satisfaction to the student and mentor whilst improving retention and completion rates.

\section{DEVELOPMENT OF THE FIRST UNIVERSITY MASTERS- LEVEL PROGRAMME IN EMERGENCY MEDICINE \& CRITICAL CARE ULTRASOUND IN THE UNITED KINGDOM}

\author{
Bob Jarman ${ }^{1}$, Simon Richards ${ }^{2}$ \\ ${ }^{1}$ Department of Emergency Medicine, Queen Elizabeth Hospital, UK \\ ${ }^{2}$ University of Teesside, UK
}

There are many established institutions in the UK offering postgraduate masters programmes in medical ultrasound, including the University of Teesside. However, with the increasing development of point-of-care ultrasound applications, especially in non-radiological specialties, there has been a need for more tailored and flexible postgraduate courses.

Traditionally, the pathway through a masters programme has been postgraduate certificate $(\mathrm{PgC})$, postgraduate diploma $(\mathrm{PgD})$ leading to Masters. The initial $\mathrm{PgC}$ level has traditionally required at least 500 scans to be performed in a mentored environment as a prerequisite of national CASE approval. This has proved difficult for practising clinicians to fulfil in addition to their normal clinical workload.

Developments in 2008/2009 centred on restructuring the medical ultrasound programme at the University of Teesside and led to a specific pathway being developed for emergency medicine and critical care ultrasound. This masters programme was awarded CASE approval in early 2009. CASE assessors understood the move towards a competency endpoint and did not define a minimum number of scans. This is the first masters level programme in the UK in emergency medicine and critical care and has been fully supported by CASE.

The ultrasound modalities covered includes shock, echocardiography, chest, hepatobiliary, urological, MSK and early pregnancy. There is flexibility of choice and competency is attained in a particular modality through completion of triggered assessments and not based on number of scans performed. The standards/level of practice of each modality is based in the College of Emergency Medicine's Level 2 Ultrasound Guidance (CEM 2008) for enhanced ultrasound. 


\section{USING OF SIMULTANEOUS CONTROLLED VIDEOS}

\section{TO TEACH ULTRASOUND ANATOMY}

Reuben Slater, Neelakantan Sunder, Lisa Warren

Department of Anesthesia and Critical Care, Massachusetts General Hospital, Boston, MA, USA

Successful performance of ultrasound guided regional anesthesia requires technical skills and knowledge of ultrasound anatomy. Simulators such as the, "Blue Phantom" allow trainees to hone technical skills. Learning about ultrasound anatomy however has been limited to viewing static images or video.

We have created a tool to enhance the teaching of ultrasound anatomy pertinent to regional anesthesia. Simultaneous videos of the probe movements and the resulting ultrasound images are obtained, illustrating anatomy relevant to regional blockade. The images are imported into Adobe Flash. The resultant software allows the videos to be manipulated with a slider. As the slider is manipulated, the images play synchronously. Backward and Forward movement of the slider simulates the kinds of movements that would be seen during live scanning.

We have produced software illustrating the neurovascular anatomy of the neck and the anatomy of the sciatic nerve in the thigh. We believe that this software will help our trainees improve their knowledge of ultrasound anatomy before performing blocks on patients. 